\title{
Prediction of Relative Stability between TACE/Gelastatin and TACE/Gelastatin Hydroxamate
}

\author{
Ky-Youb Nam, ${ }^{*}$ Gyoonhee Han, ${ }^{\dagger,}$ Hwan Mook Kim, ${ }^{\ddagger}$ and Kyoung Tai No ${ }^{\dagger,,}, \S$ \\ Research Institute Bioinformatics \& Molecular Design (BMD), Yonsei Engineering Research Complex, Seodaemun-gu, \\ Seoul 120-749, Korea. ${ }^{*}$ E-mail: kyn@bmdrc.org \\ †Department of Biotechnology, Yonsei University, Seodaemun-gu, Seoul 120-749, Korea. ${ }^{*}$ E-mail: ktno@yonsei.ac.kr \\ ${ }^{\sharp}$ Bio-Evaluation Center, Korea Research Institute of Bioscience and Biotechnology, Ochang 363-883, Korea \\ Received April 12, 2010, Accepted September 15, 2010
}

\begin{abstract}
A gelastatins (1), natural MMP inhibitors, and their hydroxamate analogues (2) in TACE enzyme evaluated for discovery of potent TACE inhibitors. We have employed molecular dynamics simulations to compute the relative free energy of hydration and binding to TACE for gelastatin (1) and its hydroxamate analogue (2). The relative free energy difference is directly described in this article using the free energy perturbation approach as a means to accurately predict the TACE inhibitor of gelastatin analogues. The results show that the good agreement between the experimental and theoretical relative free energies of binding, gelastatin hydroxamate (2) binds stronger to TACE by $-3.37 \mathrm{kcal} / \mathrm{mol}$. The desolvation energy costs significantly reduced binding affinity, hydroxamate group associated with high desolvation energy formed strong favorable interactions with TACE with more than compensated for the solvation costs and therefore led to an improvement in relative binding affinity.
\end{abstract}

Key Words: Rheumatoid Arthritis, TACE, Zn-binding anchor, Gelastatin, Free energy perturbation

\section{Introduction}

Rheumatoid Arthritis (RA) is a chronic inflammatory autoimmune disease affecting $0.5-1 \%$ of population worldwide. Typical clinical symptoms of RA are pain and joint swelling, and lead to joint destruction in long term. There have been various approaches toward improved therapeutics. ${ }^{1}$ One of attracting target is tumor necrosis factor $\alpha$ (TNF- $\alpha$ ), an important cytokine in RA pathological progress. ${ }^{2-3}$ The clinical success of antiTNF- $\alpha$ biological drugs ${ }^{4-5}$ have driven more attention to targeting TNF- $\alpha$ in RA therapy. Several "the next generation" antiTNF- $\alpha$ small molecule approaches have been in progress because of their significant advantage of developing orally active molecules in low cost. One of the targets for discovery of small molecule inhibitors of TNF- $\alpha$ is the zinc containing metalloproteinase, TNF- $\alpha$ converting enzyme (TACE, also known as ADAM-17). TACE is a structural member of the ADAM (a disintegrin and a metalloprotease) family. TACE is a crucial for the processing of several membrane-associated proteins and converts membrane bound pro-TNF- $\alpha$ to mature and soluble TNF- $\alpha .^{6-7}$

A free energy simulation technique known as the thermodynamic cycle perturbation (TCP) approach $^{8-11}$ used in conjunction with molecular dynamics calculations offers a theoretically precise method of determining the binding free energy differences of structurally related inhibitors. Despite its high accuracy, free energy calculations ${ }^{12-17}$ have primarily been used to rationalize experimentally determined binding affinities rather than predict affinities of new analogues. The reluctance to use free energy calculations for predictions and therefore drug design is partly

\footnotetext{
${ }^{\S}$ Member of Translational Research Center for Protein Function Control, Korea.

*To whom all correspondence should be addressed Tel: 82-2-3939551; Fax: 82-2-393-9554; e-mail: kyn@bmdrc.org
}

related to its large CPU requirements and its limited use for the evaluation of large sets of compounds or compounds that differ significantly in structure from the lead compound. Nevertheless, a few studies have reported promising results using the TCP approach. ${ }^{18-20}$ For example, HIV-1 protease inhibitors were successfully optimized using TCP calculations as part of an iterative structure-based design study. ${ }^{19}$ Recently, many theoretical and practical protein-ligand docking methods are suggested, X. Hu et al. ${ }^{21}$ have compared a number of docking systems and identified those that show the greatest accuracy in positioning the ligands of zinc metalloproteinases into the active site. Several scoring systems were evaluated, and knowledge-based systems were identified as the most accurate. Proper zinc coordination geometry was shown to be a prerequisite for good quality docking.

As a part of our ongoing efforts for discovery of potent TACE inhibitors, we previously evaluated gelastatins (1), natural MMP inhibitors, and their hydroxamate analogues (2) in TACE enzyme. ${ }^{22}$ In this paper, we report molecular dynamics (MD) and free energy perturbation (FEP) studies carried out on gelastatinsinhibitor complexes of TACE to obtain insights into the structural and energetic preferences of these inhibitors. Furthermore, the FEP simulations gave relative free energies of binding in good agreement with experimental results.

\section{Methods}

Model compounds. In order to investigate the relative stability between TACE when it is complexed with the inhibitor, gelastatins (1), and their hydroxamate analogues (2), ab initio MO calculations were performed. All the possible protonated states of inhibitors were taken into account as models, but the protonated states of imidazole nitrogens in hitidine residues were not considered. The TACE models can be classified into 
<smiles>C/C=C/C=C/C=C1\C=C(CCC(=O)O)C(=O)OC1</smiles>

Figure 1. Structure of ligands. The binding affinities and relative binding free energies are given in Tables 2. Note that in all cases the zinc binding group is the carboxylate and hydroxamate, generally believed to be tetrahedrally coordinated using only one oxygen of the carboxylate.

three classes: class I, Model I-Model II, has no Glu406 residue. Model I, TACE-(1) Complex Model without Glu406; Model II, TACE-(2) Complex Model without Glu406; class II, Model III, TACE-(1) Complex Model with Glu406 and protonated inhibitor(1); Model IV, TACE-(1) Complex Model with Glu406 and deprotonated inhibitor(1); Model V, TACE-(2) Complex Model with Glu406 and protonated inhibitor(2); Model VI, TACE-(2) Complex Model with Glu406 and deprotonated inhibitor(2); class III, Model VII, TACE-(1) Complex Model with Glu406 and protonated Glu406 and deprotonated inhibitor(1); Model VIII, TACE-(1) Complex Model with Glu406 and protonated Glu406 and protonated inhibitor(1); Model IX, TACE(2) Complex Model with Glu406 and protonated Glu406 and deprotonated inhibitor(2); Model X, TACE-(2) Complex Model with Glu406 and protonated Glu406 and protonated inhibitor(2); The proposed models of the TACE-inhibitor complex were simplified for the ab initio MO calculations. In the simplified models, glutamate and glutamic acid were replaced with acetic and acetic acid, respectively. The histidine residues were replaced with imidazole rings. Figure 2 shows simple model structures.

ab initio Calculations. We performed ab initio SCF calculation using Gaussian $03 .^{23}$ All geometry optimizations were performed with the B3LYP/6-31+G* basis set and the relative stability of models were calculated. The binding energy of each model in the phase $\mathrm{X}$ was calculated as follows.

$$
\begin{aligned}
& \Delta E_{\text {PhaseX }}^{\text {BindingEnergy }}=E_{\text {PhaseX }}^{\text {Model }}-\left(E_{\text {PhaseX }}^{\text {TACE,ClassI } \sim I I I}+E_{\text {PhaseX }}^{\text {Lig_Model }}\right)(\text { Eq. 1) } \\
& E_{\text {PhaseX }}^{\text {TACESI } ~} \text { and } E_{\text {PhaseX }}^{\text {Lig_Model }} \text { were calculated with geometry }
\end{aligned}
$$
using same basis set. To calculate the binding energy between simplified models in aqueous solution, self-consistent reaction fields (SCRF) calculations were performed with conductor-like polarizable continuum model (CPCM). ${ }^{24}$

Calculation of free energy difference. The free energy perturbation method was used for computing the relative free energy differences of gelastatin analogues by constructing a nonphysical path which connects the desired initial and terminal states. This approach directly enables the calculation of relative change in the free energy difference between gelastatin analogues by computationally simulating the mutation of gelastatin(1) to gelastatin hydroxamate(2). The total free energy change for the mutation from the initial to the final state is computed by summing incremental free energy changes over several windows visited by the state variable changing from 0 to 1 . The thermodynamic integration of the relative free energy assumes that the free energy change can be expressed as an integral.

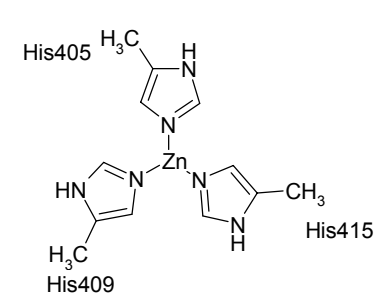

$\underset{\substack{\mathrm{O} \\ \text { Model I }}}{\mathrm{O}}$

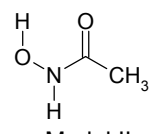

Class I

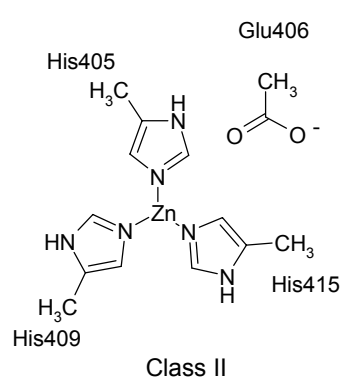

$\mathrm{H}_{\mathrm{O}} \stackrel{\mathrm{O}}{\mathrm{CH}_{3}}$

Model III

$\stackrel{\text { 兵 }}{\mathrm{CH}_{3}}$

Model IV

$\stackrel{\mathrm{O}}{\mathrm{O}} \stackrel{\mathrm{O}}{\mathrm{O}}$

$\mathrm{H}$

Model V

$\mathrm{O}_{\substack{\mathrm{N} \\ \mathrm{H}}}^{-} \stackrel{\mathrm{O}}{\mathrm{O}}$

Model VI

$\stackrel{\text { 少 }}{\mathrm{CH}_{3}}$

Model VII

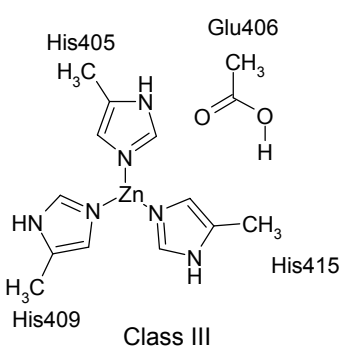

$\mathrm{H}_{\mathrm{O}} \stackrel{\mathrm{O}}{\mathrm{CH}_{3}}$

Model VIII

- - ${ }_{-\substack{\mathrm{N} \\ \mathrm{H} \\ \mathrm{H}}} \mathrm{Cl}_{\mathrm{CH}_{3}}$

Model IX

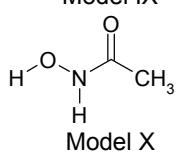

Figure 2. Sketched the active site of TACE-Gelastatin analogs complexes. The active site consists of the $\mathrm{Zn}^{++}$metal, three imidazole groups from His405, His409 and His415. Protons involved in form hydrogen bonds between the inhibitor and glutamic acid residue.

$$
\Delta A=\int_{0}^{1} \frac{\delta A(\lambda)}{\delta \lambda} d \lambda
$$

The above equation can be integrated numerically. FDTI (finite difference thermodynamic integration) ${ }^{25}$ in the Discover ${ }^{\circledR}$ program employs the perturbation formalism to numerically compute the derivatives of the free energy function, with respect to the coupling parameter $\lambda$.

$$
\begin{aligned}
\Delta A_{i} & =A\left(\lambda_{i}\right)-A\left(\lambda_{i} \pm \delta \lambda\right) \\
& =-k_{b} T \ln \left(\exp \left[-\left(E\left(\lambda_{i}\right)-E\left(\lambda_{i} \pm \delta \lambda\right)\right) / k_{b} T\right]_{i}\right)
\end{aligned}
$$

Computing $\Delta A_{i}$ for six values of $\lambda$ spanning the integral from 0.0 to 1.0 . Total free energy change $\Delta A$ can be estimated. 
(a)

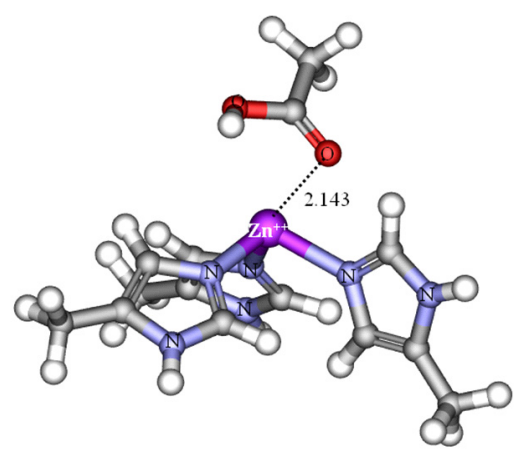

(c)

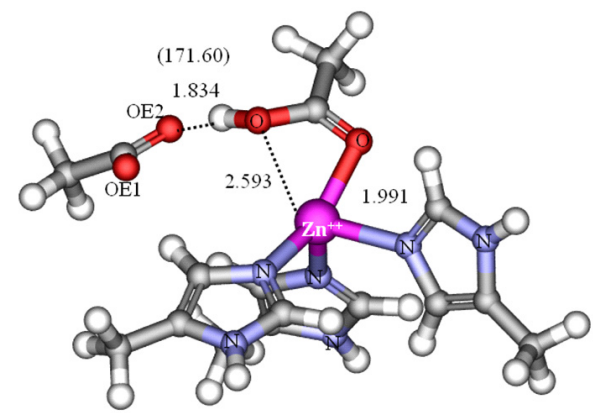

(e)

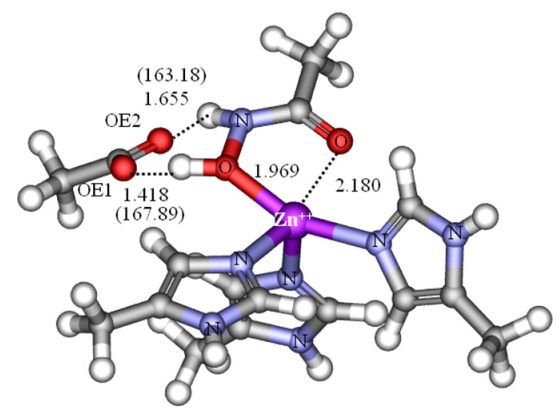

(g)

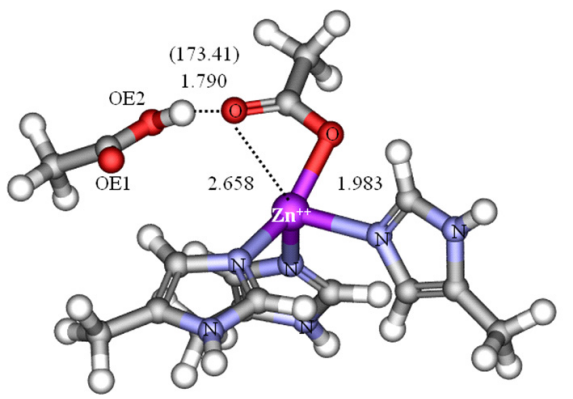

(i)

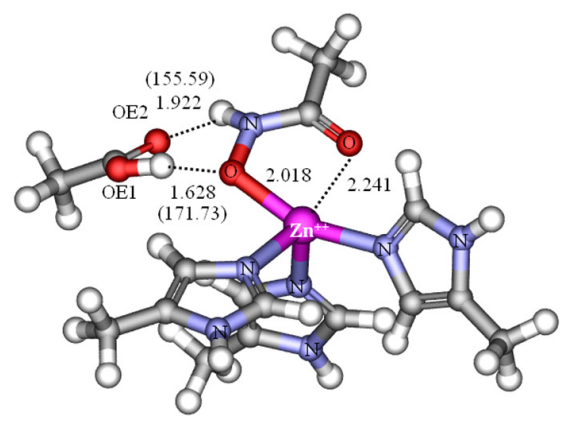

(b)

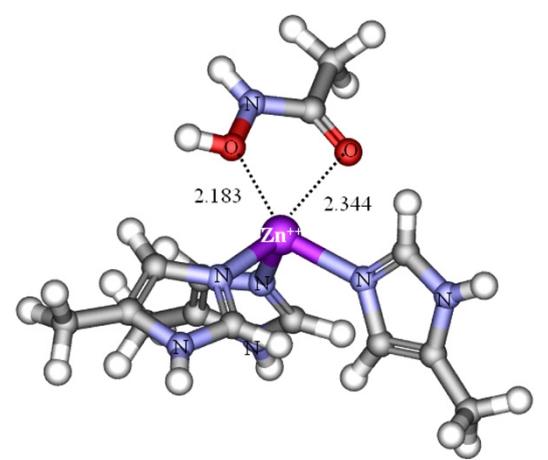

(d)

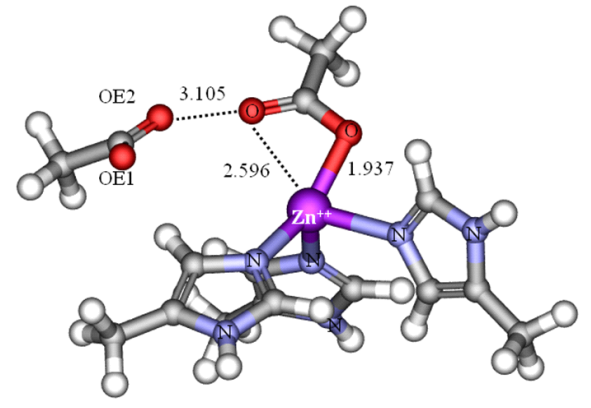

(f)

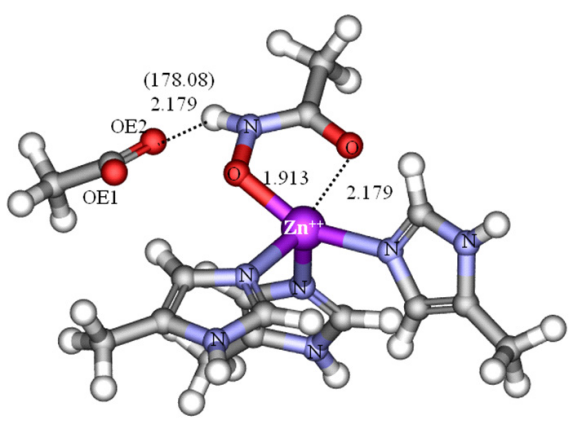

(h)

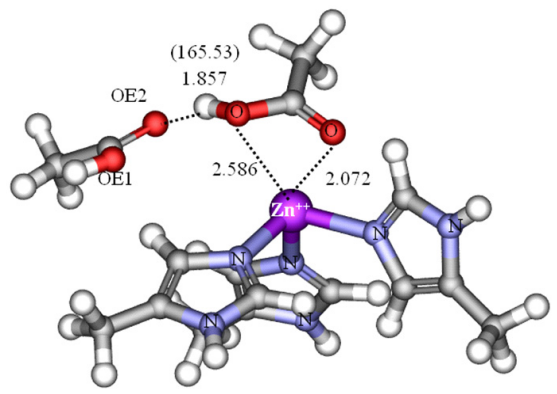

(j)

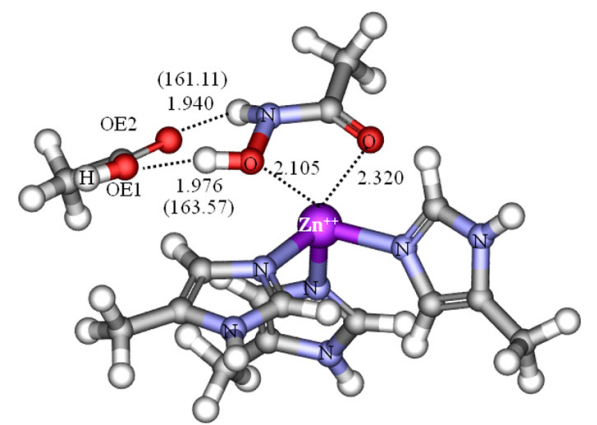

Figure 3. Optimized geometries (B3LYP/6-31+G* basis set) for the active site of TACE-inhibitor complex. The models of class I (a,b), class II $(\mathrm{c}, \mathrm{d}, \mathrm{e}, \mathrm{f})$ and class III $(\mathrm{g}, \mathrm{h}, \mathrm{I}, \mathrm{j})$. 


$$
\Delta A=-k_{b} \mathrm{~T} \sum_{i=1}^{k} \frac{\ln \left(\exp \left[-\left(E\left(\lambda_{i}\right)-E\left(\lambda_{i} \pm \delta \lambda\right)\right) / \mathrm{k}_{\mathrm{b}} \mathrm{T}\right]\right)}{\delta \lambda} \Delta \lambda_{i}
$$

All calculations were performed using the Discover 2.98/ InsightII with CVFF force field. ${ }^{26}$ The crystal structure of TACE with synthetic inhibitor was used as the computational model (1BKC.pdb). ${ }^{27}$ The TACE inhibitor 2 was built from the coordinates of a synthetic inhibitor as found in the crystal structure using Builder module in InsightII. The computational complex model was solvated using a solvent sphere of water extending $24.0 \AA$ around the catalytic zinc ion, only residues within $16.0 \AA$ of the catalytic zinc ion were allowed to move. The systems was initially minimized using 500 steps of steepest decent and 2000 steps of conjugated gradient with $14.0 \AA \AA$ nonbonded cutoff distance to eliminate the initial strain in the molecule. The zinc ion VDW radius was taken from the work of Store and Karplus. ${ }^{28}$ Numerical integration by the leap frog integrator with a $1 f_{S}$ step size was used for molecular dynamic calculation. The molecular dynamics calculations for the solvated system were performed on running at equilibrium for $30 \mathrm{ps}$, followed by $70 \mathrm{ps}$ at $300 \mathrm{~K}$. The free energy differences were collected at every 10 steps, and perturbation was used for $6 \lambda$ points.

\section{Results and Discussion}

The binding energies of the models were calculated with B3LYP/6-31+G* ab initio MO calculations and are summarized in Table 1. The model IX shows the strongest complex form. In the gas phase, models IX is relatively the most stable than the others. In the class III, Model IX is more stable than Model VII, about $-40.56 \mathrm{kcal} / \mathrm{mol}$. The binding energy of Model IX can be readily understood by comparing the optimized structures. Two hydrogen bonds and one $\mathrm{Zn}^{++}$chelating were formed in the Model IX inhibitor complex. Two hydrogen bonds are formed between the gelastatin hydroxamate (2) and TACE model (class III); i) the inhibitor oxygen and the proton on $\mathrm{OE} 1_{406}$ $\left(\mathrm{R}_{\mathrm{OH}}=1.628 \AA ; \angle \mathrm{OE} 2-\mathrm{H} . . . \mathrm{N}_{\text {inhibitor }}=171.73^{\circ}\right)$ and ii) the proton on the inhibitor-NH and OE $2_{406}\left(\mathrm{R}_{\mathrm{OH}}=1.922 \AA\right.$; $\angle \mathrm{OE} 2-$ $\left.\mathrm{H} . . . \mathrm{N}_{\text {inhibitor }}=155.59^{\circ}\right)$. There are two possible coordinations between $\mathrm{Zn}^{++}$metal and gelastatin hydroxamate (2); i) the two Oxygen in hydroxamte $\left(\mathrm{R}_{\mathrm{Zn}+\ldots \mathrm{O}}=2.018 \AA ; \mathrm{R}_{\mathrm{Zn}++\ldots \mathrm{O}}=2.241 \AA\right)$. Since all the two hydrogen bonds have suitable bond distances and bond angles for hydrogen bond formation, the Model IX can form a strong complex with gelastatin hydroxamate (2). Our previous computational results for hydrogen bond potential showed angle dependency of hydrogen bond. ${ }^{29}$ The binding energies of the models in continuum media with aqueous solution were calculated and summarized in Table I. Model IX was the most stable in the continuum environment by CPCM calculation. The problem was the binding energy of Model I, Model III, Model IV, Model VIII and Model X were positive, which implied didn't make a complex. The binding source of gelastatin is not only good chelating but also hydrogen bond. We suggest the TACE model with inhibitor must be considerd the effects of the side-chain of Glu406 for relative complex energies. Finally, Model VII shows the stable binding energy, $-23.74 \mathrm{kcal} / \mathrm{mol}$.

To include the influence of the hydrogen bonds between the model and the water and the structural change of the water by the model, molecular dynamic simulations were performed with explicit water molecules. When calculating relative free energy differences, a thermodynamic cycle is generally applied. ${ }^{30-31}$ Here, we have used a thermodynamic cycle, as exhibited in Figure 5. Since the free energy is a thermodynamic state function, the left and right sides of the cycle give the relative free energy of hydration,

$$
\Delta \Delta A_{\text {hyd }}=\Delta A_{2}-\Delta A_{1}=\Delta A_{t r}^{\text {sol }}-\Delta A_{t r}^{\text {gas }}
$$

and the free energy of binding, repectively.

$$
\Delta \Delta A_{\text {bind }}=\Delta A_{4}-\Delta A_{3}=\Delta A_{t r}^{\text {hyd }, \text { comp }}-\Delta A_{t r}^{\text {sol }}
$$

Because the quantities $\left(\Delta A_{h y d}\right)$ and $\left(\Delta A_{\text {bind }}\right)$ are impracticable to compute, $\Delta \Delta A_{\text {hyd }}$ and $\Delta \Delta A_{\text {bind }}$ are obtained through the simulation of a nonphysical process in three different environments. In our case the mutation of gelastatin (1) into gelastatin hydroxamate (2) in the gas phase, in solution and inside the solvated

\begin{tabular}{|c|c|c|c|c|c|c|}
\hline & $\begin{array}{l}E_{\text {gas }}^{T A C E, \text { Class } ~} \sim I I I \\
\text { (Hartree) }\end{array}$ & $\begin{array}{l}E_{\text {gas }}^{\text {Lig_Model }} \\
\text { (Hartree) }\end{array}$ & $\begin{array}{l}\text { Binding Energy } \\
(\mathrm{kcal} / \mathrm{mol})\end{array}$ & $\begin{array}{l}E_{\text {aqueous }}^{\text {TACE,ClassI III }} \\
\text { (Hartree) }\end{array}$ & $\begin{array}{l}E_{\text {aqueous }}^{\text {Lig_Model }} \\
\text { (Hartree) }\end{array}$ & $\begin{array}{l}\text { Binding Energy } \\
(\mathrm{kcal} / \mathrm{mol})\end{array}$ \\
\hline Model I & -2575.370 & -229.094 & -23.834 & -2575.625 & -229.111 & 9.815 \\
\hline Model II & -2575.370 & -284.368 & -48.458 & -2575.625 & -284.404 & -8.892 \\
\hline Model III & -2804.111 & -229.094 & -58.678 & -2804.315 & -229.111 & 1.867 \\
\hline Model IV & -2804.111 & -228.539 & -124.209 & -2804.315 & -228.649 & 10.624 \\
\hline Model V & -2804.111 & -284.368 & -110.494 & -2804.315 & -284.404 & -7.469 \\
\hline Model VI & -2804.111 & -283.811 & -162.465 & -2804.315 & -283.923 & -11.899 \\
\hline Model VII & -2804.468 & -228.539 & -195.807 & -2804.735 & -228.649 & -23.743 \\
\hline Model VIII & -2804.468 & -229.094 & -39.478 & -2804.735 & -229.111 & 3.948 \\
\hline Model IX & -2804.468 & -283.811 & -236.363 & -2804.735 & -283.923 & -54.505 \\
\hline Model X & -2804.468 & -284.368 & -56.722 & -2804.735 & -284.404 & 0.195 \\
\hline
\end{tabular}
complex, gives $\left(\Delta A_{t r}^{\text {sol }}\right),\left(\Delta A_{t r}^{\text {gas }}\right)$, and $\left(\Delta A_{t r}^{\text {hyd }, \text { comp }}\right)$, respectively.

Table 1. Binding energy for TACE-inhibitor complexes $(1$ Hartree $=627.5095 \mathrm{kcal} / \mathrm{mol})$ 
Table 2. Calculate relative solvation and binding free energies between gelastatin (1) and its analog (2) to TACE (unit are in $\mathrm{kcal} / \mathrm{mol}$ )

\begin{tabular}{lc}
\hline \multicolumn{1}{c}{ Transfer } & $1 \rightarrow 2$ \\
\hline$\Delta A_{t r}^{\text {gas }}$ & $5.43 \pm 0.05$ \\
$\Delta A_{t r}^{\text {sol }}$ & $9.40 \pm 0.16$ \\
$\Delta A_{t r}^{\text {hyd,comp }}$ & $6.03 \pm 0.35$ \\
$\Delta A_{t r}^{\text {gas,comp } a}$ & $-6.48 \pm 2.64$ \\
$\Delta \Delta A_{\text {hyd }}$ & $3.97 \pm 0.21^{b}$ \\
$\Delta \Delta A_{\text {bind }}^{\text {cal }}$ & $-3.37 \pm 0.51^{b}$ \\
$\Delta \Delta A_{\text {bind }}^{\text {exp }}$ & -2.54
\end{tabular}

${ }^{a}$ The binding free energy at the gas phase $\left(\Delta A_{t r}^{\text {gas,comp }}\right)$ calculate from transfer energy from TACE$\cdot(2) \rightarrow \mathrm{TACE} \cdot(1) .{ }^{b}$ The reported errors were obtained com-puting sum of two state errors. ${ }^{c} \Delta \Delta A_{\text {bind }}^{\text {exp }}$ approximately calculated from $\begin{aligned} \Delta \Delta A_{\text {bin }}^{\text {exp }}=-2.303 R T \log \left(\frac{I C_{50}^{(2)}}{I C_{50}^{(1)}}\right) & =-2.303 R T \log (1.98 \mu \mathrm{M} / 0.028 \mu \mathrm{M}) \\ & =-2.54 \mathrm{kcal} / \mathrm{mol} .\end{aligned}$

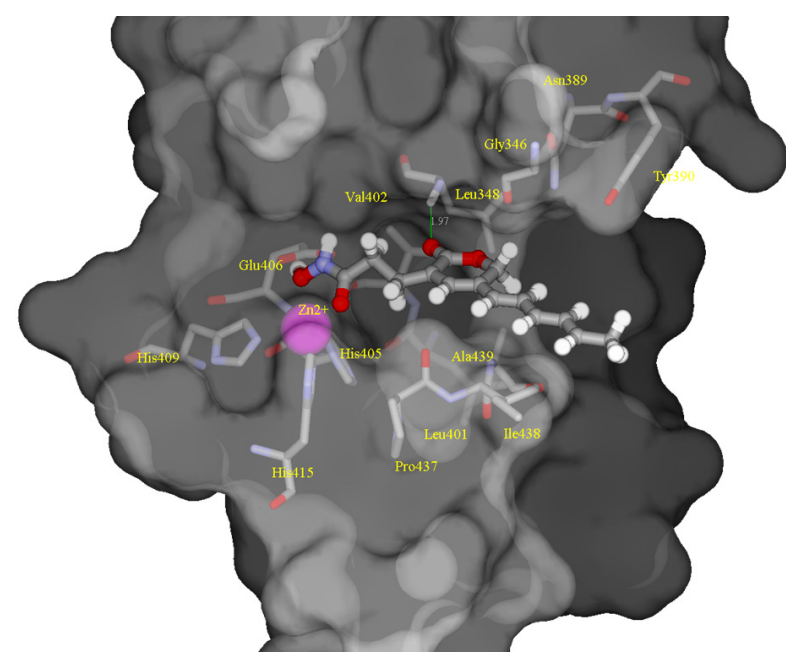

Figure 4. Orientation of zinc in active site. The coordination of the catalytic zinc in a representative molecular dynamics structure with the ligand 2 is shown, along with the position of glu 406 . Note that only the zinc binding anchor of the ligand 2 is shown. In the ligand 2 trajectory, another crucial hydrogen bond forms between leu338 backbone $\mathrm{N}-\mathrm{H}$ and an oxygen of the ligand 2 within $1.97 \AA$.

This mutation involves the perturbation of a acetic acid group into a hydroxmate group. Experimental results in the TACE inhibition show that gelastatins hydroxamate (2) binds more strongly to TACE than gelastatin (1) by approximately -2.54 $\mathrm{kcal} / \mathrm{mol}$, it is not directly experimental free energy but indication of free energy difference. The experimental and calculated results (Table 2) show that gelastatin hydroxamate (2) has a higher affinity for TACE than gelastatin (1). The calculated difference in binding free energy of $-3.37 \pm 0.51 \mathrm{kcal} / \mathrm{mol}$ is in good agreement with the experimentally measure difference of -2.54 $\mathrm{kcal} / \mathrm{mol}$. Jorgensen groups ${ }^{32}$ suggested that inhibitors with a higher dipole moment bind more strongly to water than to enzyme, so lowering the affinity constant. Here, we calculate the difference of hydration free energy $\left(\Delta \Delta A_{\text {hyd }}\right)$ that gelastatin (1) is more solvated than gelastatin hydroxamate (2) by $3.97 \mathrm{kcal} /$

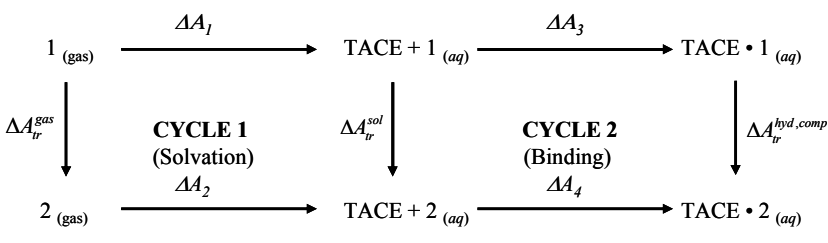

Figure 5. Thermodynamic cycles used to calculate the relative solvation energy (cycle 1) and relative binding free energy (cycle 2).

mol. In addition, gelastatin hydroxamate (2) is associated with increased conformational freedom (higher entropy) relative to gelastatin (1) and therefore likely pays greater entropic costs. However, the binding free energy ( $\Delta A_{t r}^{\text {hyd,comp }}$ ) of gelastatin hydroxamate (2) to TACE is stronger than the binding of gelastatin (1) by $-3.37 \mathrm{kcal} / \mathrm{mol}$, indicating that gelastatin hydroxmate has a net interaction of $-6.48 \mathrm{kcal} / \mathrm{mol}\left(\Delta A_{t r}^{\text {gas,comp }}\right)$ with TACE and can be overcome the desolvation penalty $\left(\Delta \Delta A_{\text {hyd }}\right)$, respectively. Figure 4 shows that hydroxamate group of gelastatin hydroxamate(2) is mainly chelated to $\mathrm{Zn}^{2+}$ ion and additionally form a hydrogen bond to side chain of Glu406. This suggests that the additional interaction of gelastatin hydroxamate (2) to TACE has a strong electrostatic character, which is absent in gelastatin (1).

\section{Conclusions}

In this work, we employed ab initio calculations and molecular dynamics simulations to compute the relative free energy of hydration and binding to TACE for gelastatin (1) and its hydroxamate analogue (2). The binding energy shows the Model IX is the most stable and the relative free energy difference is directly described in this article using the FEP approach as a means to accurately predict the TACE inhibitor of gelastatin analogues. The results show that the good agreement between the experimental and theoretical relative free energies of binding, despite being more solvated than gelastatin (1) by $\Delta \Delta A_{\text {hyd }}$, $3.97 \mathrm{kcal} / \mathrm{mol}$, gelastatin hydroxamate (2) binds stronger to TACE by $\Delta \Delta A_{\text {bind }}^{\text {cal }},-3.37 \mathrm{kcal} / \mathrm{mol}$ (Table 2 ). The desolvation energy costs significantly reduced binding affinity, hydroxamate group associated with high desolvation energy formed strong favorable interactions with TACE with more than compensated for the solvation costs and therefore led to an improvement in relative binding affinity. Accurate prediction of inhibitors is expected to shorten the time required to find suitable candidates for drug discovery by eliminating the time expended on the synthesis. Except for the FEP method, which is very expensive computationally and cannot be used for all proposed analogues of a lead compound, no other computation method provides a accurate solvation free energies.

Acknowledgments. This research was supported by Futurebased Technology Development Program, through the National Research Foundation of Korea(NRF) funded by the Ministry of Education, Science and Technology (2010-0018164) and Ministry for Health, Welfare \& Family Affairs (A101836-10110000600) Republic of Korea. 


\section{References}

1. Smolen, J. S.; Steiner, G. Nature Rev. Drug Discovery 2003, 2, 473-488.

2. Newton, R. C.; Decicco, C. P. J. Med. Chem. 1999, 42, 2295-2314.

3. Palladino, M. A.; Bahjat, F. R.; Theodorakis, E. A.; Moldawer, L. L. Nature Rev. Drug Discovery 2003, 2, 736-746.

4. Moreland, L. W.; Baumgartner, S. W.; Schiff, M. H.; Tindall, E. A.; Fleischmann, R. M.; Weaver, A. L. et al. N. Engl. J. Med. 1997, 337, 141-147.

5. Lipsky, P. E.; van der Heijde, D. M.; ST Clair, W. E.; Furst, D. E.; Breedveld, F. C.; Kalden, J. R. et al. N. Engl. J. Med. 2000, 343, 1594-1602.

6. Black, R. A.; Rauch, C. T.; Kozlosky, C. J.; Peschon, J. J.; Slack, J. L.; Wolfson, M. F. et al. Nature 1997, 385, 729-733.

7. Moss, M. L.; Jin, S. C.; Milla, M. E.; Burkhart, W.; Carter, H. L.; Chen, W.-J. et al. Nature 1997, 385, 733-736.

8. Beveridge, D. L.; DiCapua, F. M. Annu. Rev. Biophys. Biophys. Chem. 1989, 18, 431-492.

9. McCammon, J. A. Curr. Opin. Struct. Biol. 1991, 1, 196-200.

10. Rami, M. R.; Bacquet, R. J.; Zichi, D.; Matthews, D. A.; Welsh, K. M.; Jones, T. R. et al. J. Am. Chem. Soc. 1992, 114, 10117-10122.

11. Gao, J.; Kuczera, K.; Tidor, B.; Karplus, M. Science 1989, 244 , 1069-1072.

12. Reddy, M. R.; Erion, M. D.; Agarwal A. In Reviews in Computational Chemistry; Lipkowitz, K. B., Boyd, D. B., Eds.; WileyVCH: New York, USA, 2000; Vol. 16, pp 217-304.

13. Reddy, M. R.; Viswanadhan, V. N.; Weinstein, J. N. Proc. Natl. Acad. Sci. USA 1991, 88, 10287-10291.

14. Ferguson, D. M.; Radmer, R. J.; Kollman, P. A. J. Med. Chem. 1991, 34, 2654-2659.

15. Tropsha, A. J.; Hermans, J. Protein Eng. 1992, 5, 29-33.

16. Rao, B. G.; Tilton, R. F.; Singh, U. C. J. Am. Chem. Soc. 1992, 114,
4447-52.

17. Erion, M. D.; Reddy, M. R. J. Am. Chem. Soc. 1998, 120, 32953304.

18. Merz, K. M.; Kollman, P. A. J. Am. Chem. Soc. 1989, 111, 5649-58.

19. Reddy, M. R.; Varney, M. D.; Kalish, V.; Viswanadhan, V. N.; Appelt, K. J. Med. Chem. 1994, 37, 1145-52.

20. Nam, K.-Y.; Chang, B. H.; Han, C. K.; Ahn, S. K.; No, K. T. Bull. Korean Chem. Soc. 2003, 24, 817-823.

21. Hu, X.; Balaz, S.; Shelver, W. H. J. of Mol. Graph. \& Model. 2004, 22, 293-307.

22. Kim, H. M.; Choi, H.-M.; Tae, H. S.; Kim, B. G.; Lee, H.-Y.; Nam, K.-Y. et al. Biochem. \& Biophys. Res. Commun. 2006, 341, 627634.

23. Frisch, M. J. et al. GAUSSIAN 03, Revision B2; Gaussian Inc.: Pittsburgh, PA, 2003.

24. Klamt, A.; Schüürmann, G. J. Chem. Soc., Perkin Trans. 1993, 2, 799-805

25. Mezei, M. J. Chem. Phys. 1987, 86, 7084-7088.

26. Hagler, A. T.; Lifson, S.; Dauber, P. J. Am. Chem. Soc. 1979, 101, 5111-5121.

27. Maskos, K.; Fernandez-Catalan, C.; Huber, R.; Bourenkov, G. P.; Bartunik, H.; Ellestad, G. A. et al. Proc. Natl. Acad. Sci. USA 1998, 95, 3408-3412.

28. Stote, R. H.; Karplus, M. Proteins: Strut., Funct. and Gent. 1995 , 23, 12-31.

29. No, K. T.; Kwon, O. Y.; Kim, S. Y.; Jhon, M. S.; Scheraga, A. H. J. Phys. Chem. 1995, 99, 3478.

30. van Gunsteren, W. F.; Mark, A. E. Eur. J. Biochem. 1992, 204, 947961.

31. Beveridge, D. L.; DiCapua, F. M. Annu. Rev. Biophys. Biophys. Chem. 1989, 18, 431-492.

32. Essex, J. W.; Severance, D. L.; Tirado-Rives, J.; Jorgensen, W. L. J. Phys. Chem. B 1997, 101, 9663-9669. 